\title{
Almost Periodic Solution for Memristive Neural Networks with Time-Varying Delays
}

\author{
Huaiqin Wu and Luying Zhang \\ Department of Applied Mathematics, Yanshan University, Qinhuangdao 066004, China \\ Correspondence should be addressed to Huaiqin Wu; huaiqinwu@ysu.edu.cn
}

Received 28 January 2013; Accepted 8 April 2013

Academic Editor: Maoan Han

Copyright (c) $2013 \mathrm{H}$. Wu and L. Zhang. This is an open access article distributed under the Creative Commons Attribution License, which permits unrestricted use, distribution, and reproduction in any medium, provided the original work is properly cited.

\begin{abstract}
This paper is concerned with the dynamical stability analysis for almost periodic solution of memristive neural networks with time-varying delays. Under the framework of Filippov solutions, by applying the inequality analysis techniques, the existence and asymptotically almost periodic behavior of solutions are discussed. Based on the differential inclusions theory and Lyapunov functional approach, the stability issues of almost periodic solution are investigated, and a sufficient condition for the existence, uniqueness, and global exponential stability of the almost periodic solution is established. Moreover, as a special case, the condition which ensures the global exponential stability of a unique periodic solution is also presented for the considered memristive neural networks. Two examples are given to illustrate the validity of the theoretical results.
\end{abstract}

\section{Introduction}

Memristor (resistor with memory), which was firstly postulated by Chua in [1], is the fourth fundamental electronic component along with the resistor, inductor, and capacitor. On May 1, 2008, the Hewlett-Packard (HP) research team announced their realization of a memristor prototype, with an official publication in Nature $[2,3]$. This new circuit element is a two-terminal element, either a charge-controlled memristor or a flux-controlled memristor, and shares many properties of resistors and the same unit of measurement (ohm). Subsequently, memristor has received a great deal of attention from many scientists because of its potential applications in next generation computer and powerful brainlike "neural" computer [4-14].

Recently, various memristor-based networks have been established by means of the memristive circuits, and many applications have been made in science and engineering fields [15-17]; see, for example, Cserey et al. who presented simulation measurements of a memristor crossbar device and designed a PCB memristor package and the appropriate measurement board [17]. It should be pointed that, in many applications, the existing memristor-based networks which many researchers had designed have been found to be computationally restrictive.
Neural networks, such as Hopfield neural networks, Cellular neural networks, Cohen-Grossberg, and bidirectional associative neural networks, are very important nonlinear circuit networks, and, in the past few decades, have been extensively studied due to their potential applications in classification, signal and image processing, parallel computing, associate memories, optimization, cryptography, and so forth. [18-27]. Many results, which deal with the dynamics of various neural networks such as stability, periodic oscillation, bifurcation, and chaos, have been obtained by applying Lyapunov stability theory; see, for example [28-45] and the references therein. Very recently, memristor-based neural networks (memristive neural networks) have been designed by replacing the resistors in the primitive neural networks with memristors in [46-52]. As is well known, the memristor exhibits the feature of pinched hysteresis, which means that a lag occurs between the application and the removal of a field and its subsequent effect, just as the neurons in the human brain have. Because of this feature, the memristive neural networks can remember its past dynamical history, store a continuous set of states, and be "plastic" according to the presynaptic and postsynaptic neuronal activity. In [46], Itoh and Chua designed a memristor cellular automaton and a memristor discrete-time cellular neural network, which can 
perform a number of applications such as logical operations, image processing operations, complex behaviors, higher brain functions, and RSA algorithm. In [47], Pershin and Di Ventra constructed a simple neural network consisting of three electronic neurons connected by two memristoremulator synapses and demonstrated experimentally the formation of associative memory in these memristive neural networks. This experimental demonstration opens up new possibilities in the understanding of neural processes using memory devices, an important step forward to reproduce complex learning, adaptive and spontaneous behavior with electronic neural networks.

It is well known that, in the design of practical neural networks, the qualitative analysis of neural network dynamics plays an important role. For example, to solve problems of optimization, neural control, and signal processing, neural networks have to be designed in such a way that, for a given external input, they exhibit only one globally asymptotically/exponentially stable equilibrium point. Hence, in practice applications, it is an essential issue to discuss the stability for the memristive neural networks. In [48], Hu and Wang proposed a piecewise-linear mathematical model of the memristor to characterize the pinched hysteresis feature. Based on this model, the memristor-based recurrent neural network model with time delays was given and two sufficient conditions for the global uniform asymptotic stability of the memristor-based recurrent neural networks were obtained. In $[49,50]$, Wu et al. investigated the synchronization control issue of a general class of memristor-based recurrent neural networks with time delays, and some sufficient conditions were obtained to guarantee the exponential synchronization of the coupled networks based on drive-response concept. In [51], the dynamic behaviors for a class of memristorbased Hopfield networks were analyzed, and some sufficient conditions were obtained to ensure the essential bound of solutions and global exponential stability. In [52], the stability was considered for the memristor-based recurrent network with bounded activation functions and bounded time-varying delays in the presence of strong external stimuli, and a sufficient condition on the bounds of stimuli was derived for global exponential stability of memristor-based recurrent networks.

It should be noted that, very little attention has been paid to dealing with the periodicity issue, in particular; to the best of our knowledge, the almost periodic dynamics of memristive neural networks with time-varying delays have never been considered in the previous literature, which motivates the work of this paper.

In this paper, our aim is to study the exponential stability of almost periodic solution for memristive neural networks with time-varying delays. By using the concept of Filippov solutions for the differential equations with discontinuous right-hand sides and the inequality analysis techniques, the existence and asymptotically almost periodic behavior of solution will be discussed. Based on the differential inclusions theory, the proof of the existence of the almost periodic solution will be given. By applying Lyapunov functional approach, a sufficient condition will be established to ensure the uniqueness and global exponential stability of the almost periodic solution for the considered memristive neural networks. As a special case, the conditions of the global exponential stability of a unique periodic solution equilibrium point are also presented.

The rest of this paper is organized as follows. In Section 2, the model formulation and some preliminaries are given. In Section 3, the existence and asymptotically almost periodic behavior of solutions are analyzed, the existence of the almost periodic solution is proved, and the uniqueness and global exponential stability of the almost periodic solution are investigated. In Section 4, two numerical examples are presented to demonstrate the validity of the proposed results. Some conclusions are made in Section 5.

Notations. Throughout this paper, $R$ denotes the set of real numbers, $R^{n}$ denotes the $n$-dimensional Euclidean space, and $R^{m \times n}$ denotes the set of all $m \times n$ real matrices. For any matrix $A, A^{T}$ denotes the transpose of $A$. If $A$ is a real symmetric matrix, $A>0(A<0)$ means that $A$ is positive definite (negative definite). Given the column vectors $x=$ $\left(x_{1}, \ldots, x_{n}\right)^{T}, y=\left(y_{1}, \ldots, y_{n}\right)^{T} \in R^{n}, x^{T} y=\sum_{i=1}^{n} x_{i} y_{i},|x|=$ $\left(\left|x_{1}\right|, \ldots,\left|x_{n}\right|\right)^{T}$, and $\|x\|=\left(\sum_{i=1}^{n} x_{i}^{2}\right)^{1 / 2} \cdot\|A\|=\sqrt{\lambda_{M}\left(A^{T} A\right)}$ represents the norm of $A$, where $\lambda_{M}(A)$ is the maximum eigenvalue of $A . C\left([-\tau, 0] ; R^{n}\right)$ denotes the family of continuous function $\varphi$ from $[-r, 0]$ to $R^{n}$ with the norm $\|\varphi\|=$ $\sup _{-r \leq s \leq 0}|\varphi(s)| . \dot{x}(t)$ denotes the derivative of $x(t)$. Matrices, if their dimensions are not explicitly stated, are assumed to have compatible dimensions for algebraic operations.

\section{Model Description and Preliminaries}

The KCL equation of the $i$ th subsystem of a general class of neural networks with time-varying delays can be written as

$$
\begin{aligned}
\dot{x}_{i}(t)= & -\frac{1}{\mathbb{C}_{i}}\left[\sum_{j=1}^{n}\left(\frac{1}{\mathbb{R}_{i j}}+\frac{1}{\mathbb{F}_{i j}}\right) \times \operatorname{sgn}_{i j}+\frac{1}{\mathscr{R}_{i}}\right] x_{i}(t) \\
& +\frac{1}{\mathbb{C}_{i}} \sum_{j=1}^{n} \frac{g_{j}\left(x_{j}(t)\right)}{\mathbb{R}_{i j}} \times \operatorname{sgn}_{i j} \\
& +\frac{1}{\mathbb{C}_{i}} \sum_{j=1}^{n} \frac{g_{j}\left(x_{j}\left(t-\tau_{j}(t)\right)\right)}{\mathbb{F}_{i j}} \times \operatorname{sgn}_{i j} \\
& +\frac{\mathbb{\square}_{i}}{\mathbb{C}_{i}}, \quad t \geq 0, i=1,2, \ldots, n,
\end{aligned}
$$

where $x_{i}(t)$ is the voltage of the capacitor $\mathbb{C}_{i} ; \mathbb{R}_{i j}$ denotes the resistor between the feedback function $g_{i}\left(x_{i}(t)\right)$ and $x_{i}(t) ; \mathbb{F}_{i j}$ denotes the resistor between the feedback function $g_{i}\left(x_{i}(t-\right.$ $\left.\left.\tau_{i}(t)\right)\right)$ and $x_{i}(t) ; \tau_{i}(t)$ corresponds to the transmission delay; $\mathscr{R}_{i}$ represents the parallel resistor corresponding to the capacitor $\mathbb{C}_{i} ; \mathbb{\square}_{i}$ is the external input or bias;

$$
\operatorname{sgn}_{i j}= \begin{cases}1, & i \neq j, \\ -1, & i=j .\end{cases}
$$


Let $\widetilde{d}_{i}=-\left(1 / \mathbb{C}_{i}\right)\left[\sum_{j=1}^{n}\left(1 / \mathbb{R}_{i j}+1 / \mathbb{F}_{i j}\right) \times \operatorname{sgn}_{i j}+1 / \mathscr{R}_{i}\right], \widetilde{a}_{i j}=$ $\operatorname{sgn}_{i j} / \mathbb{C}_{i} \mathbb{R}_{i j}, \widetilde{b}_{i j}=\operatorname{sgn}_{i j} / \mathbb{C}_{i} \mathbb{F}_{i j}$, and $I_{i}=\mathbb{q}_{i} / \mathbb{C}_{i}$, then (1) can be rewritten as

$$
\begin{array}{r}
\dot{x}_{i}(t)=-\widetilde{d}_{i} x_{i}(t)+\sum_{j=1}^{n} \widetilde{a}_{i j} g_{j}\left(x_{j}(t)\right) \\
+\sum_{j=1}^{n} \widetilde{b}_{i j} g_{j}\left(x_{j}\left(t-\tau_{j}(t)\right)\right)+I_{i}, \\
t \geq 0, i=1,2, \ldots, n .
\end{array}
$$

By replacing the resistors $\mathbb{R}_{i j}$ and $\mathbb{F}_{i j}$ in the primitive neural networks (1) or (3) with memristors whose memductances are $\mathbb{W}_{i j}$ and $\mathbb{M}_{i j}$, respectively, then memristive neural networks with time-varying delays can be designed as

$$
\begin{gathered}
\dot{x}_{i}(t)=-d_{i}\left(x_{i}(t)\right) x_{i}(t)+\sum_{j=1}^{n} a_{i j}\left(x_{i}(t)\right) g_{j}\left(x_{j}(t)\right) \\
+\sum_{j=1}^{n} b_{i j}\left(x_{i}(t)\right) g_{j}\left(x_{j}\left(t-\tau_{j}(t)\right)\right)+I_{i}, \\
t \geq 0, i=1,2, \ldots, n,
\end{gathered}
$$

where $d_{i}\left(x_{i}(t)\right)=-\left(1 / \mathbb{C}_{i}\right)\left[\sum_{j=1}^{n}\left(\mathbb{W}_{i j}+\mathbb{M}_{i j}\right) \times \operatorname{sgn}_{i j}+1 / \mathscr{R}_{i}\right]$, $a_{i j}\left(x_{i}(t)\right)=\left(\mathbb{W}_{i j} / \mathbb{C}_{i}\right) \times \operatorname{sgn}_{i j}, b_{i j}\left(x_{i}(t)\right)=\left(\mathbb{M}_{i j} / \mathbb{C}_{i}\right) \times \operatorname{sgn}_{i j}$, and $I_{i}=\square_{i} / \mathbb{C}_{i}$.

Combining the typical current-voltage characteristics of memristor (see Figure 1 in [48]), similarly to discussion in $[49,50]$, the coefficient parameters of the system $(4) d_{i}\left(x_{i}(t)\right)$, $a_{i j}\left(x_{i}(t)\right)$, and $b_{i j}\left(x_{i}(t)\right)$ can be modeled as

$$
\begin{aligned}
& d_{i}\left(x_{i}(t)\right)= \begin{cases}\widehat{d}_{i}, & \left|x_{i}(t)\right|<T_{i}, \\
\tilde{d}_{i}, & \left|x_{i}(t)\right|>T_{i},\end{cases} \\
& a_{i j}\left(x_{i}(t)\right)= \begin{cases}\widehat{a}_{i j}, & \left|x_{i}(t)\right|<T_{i}, \\
\check{a}_{i j}, & \left|x_{i}(t)\right|>T_{i},\end{cases} \\
& b_{i j}\left(x_{i}(t)\right)= \begin{cases}\widehat{b}_{i j}, & \left|x_{i}(t)\right|<T_{i}, \\
\check{b}_{i j}, & \left|x_{i}(t)\right|>T_{i},\end{cases}
\end{aligned}
$$

where switching jumps $T_{i}>0, \widehat{d}_{i}>0, \check{d}_{i}>0, \widehat{a}_{i j}, \check{a}_{i j}, \widehat{b}_{i j}$, and $\check{b}_{i j}, i, j=1,2, \ldots, n$, are constant numbers.

The initial value associated with the system (4) is $x_{i}(t)=$ $\varphi_{i}(t) \in C([-\tau, 0] ; R), i=1,2, \ldots, n$.

Let $\bar{d}_{i}=\max \left\{\widehat{d}_{i}, \breve{d}_{i}\right\}, \underline{d}_{i}=\min \left\{\widehat{d}_{i}, \breve{d}_{i}\right\}, \bar{a}_{i j}=\max \left\{\widehat{a}_{i j}, \breve{a}_{i j}\right\}$, $\underline{a}_{i j}=\min \left\{\widehat{a}_{i j}, \breve{a}_{i j}\right\}, \bar{b}_{i j}=\max \left\{\widehat{b}_{i j}, \breve{b}_{i j}\right\}$, and $\underline{b}_{i j}=\min \left\{\widehat{b}_{i j}, \breve{b}_{i j}\right\}$. Notice that the system (4) is a differential equation with discontinuous right-hand sides, and based on the theory of differential inclusions [53], if $x_{i}(t)$ is a solution of (4) in the sense of Filippov [54], then

$$
\begin{array}{r}
\dot{x}_{i}(t) \in-\left[\underline{d}_{i}, \bar{d}_{i}\right] x_{i}(t)+\sum_{j=1}^{n}\left[\underline{a}_{i j}, \bar{a}_{i j}\right] g_{j}\left(x_{j}(t)\right) \\
+\sum_{j=1}^{n}\left[\underline{b}_{i j}, \bar{b}_{i j}\right] g_{j}\left(x_{j}\left(t-\tau_{j}(t)\right)\right)+I_{i}(t), \\
t \geq 0, i=1,2, \ldots, n .
\end{array}
$$

The differential inclusion system (6) can be transformed into the vector form as

$$
\begin{aligned}
\dot{x}(t) \in- & {[\underline{D}, \bar{D}] x(t)+[\underline{A}, \bar{A}] g(x(t)) } \\
& +[\underline{B}, \bar{B}] g(x(t-\tau(t)))+I(t), \quad t \geq 0,
\end{aligned}
$$

where $\underline{D}=\operatorname{diag}\left(\underline{d}_{1}, \underline{d}_{2}, \ldots, \underline{d}_{n}\right), \bar{D}=\operatorname{diag}\left(\bar{d}_{1}, \bar{d}_{2}, \ldots, \bar{d}_{n}\right), \bar{A}=$ $\left(\bar{a}_{i j}\right)_{n \times n}, \underline{A}=\left(\underline{a}_{i j}\right)_{n \times n}, \bar{B}=\left(\bar{b}_{i j}\right)_{n \times n}$, and $\underline{B}=\left(\underline{b}_{i j}\right)_{n \times n}$. Or equivalently, there exist measurable functions $\mathscr{D}(t) \in[\underline{D}, \bar{D}]$, $\mathscr{A}(t) \in[\underline{A}, \bar{A}]$, and $\mathscr{B}(t) \in[\underline{B}, \bar{B}]$, such that

$$
\begin{aligned}
\dot{x}(t)= & -\mathscr{D}(t) x(t)+\mathscr{A}(t) g(x(t)) \\
& +\mathscr{B}(t) g(x(t-\tau(t)))+I(t), \quad t \geq 0 .
\end{aligned}
$$

To obtain the main results of this paper, some definitions and lemmas are introduced as follows.

Definition 1 (see [55]). A continuous function $x(t): R \rightarrow R^{n}$ is said to be almost periodic on $R$, if, for any $\varepsilon>0$, there exists a scalar $l=l(\varepsilon)>0$ and, for any interval with length $l$, there exists a scalar $\omega=\omega(\varepsilon)>0$ in this interval, such that $\|x(t+\omega)-x(t)\|<\varepsilon$ for all $t \in R$.

Definition 2. The almost periodic solution $x^{*}(t)$ of the system (4) is said to be globally exponentially stable, if there exist scalars $\eta>0$ and $\delta>0$, such that

$$
\left\|x(t)-x^{*}(t)\right\| \leq \eta e^{-\delta t}, \quad t \geq 0,
$$

where $x(t)$ is the solution of the system (4) with the initial value $x(t)=\varphi(t) \in C\left([-\tau, 0] ; R^{n}\right) . \delta$ is called as the exponential convergence rate.

Definition 3 (see [55]). The solution $x(t)$ of the system (4) with the initial value $x(t)=\varphi(t) \in C\left([-\tau, 0] ; R^{n}\right)$ is said to be asymptotically almost periodic, if, for any $\varepsilon>0$, there exist scalars $T>0, l=l(\varepsilon)>0$, and $\omega=\omega(\varepsilon)>0$ in any interval with length $l$, such that $\|x(t+\omega)-x(t)\|<\varepsilon$, for all $t \geq T$.

Lemma 4 (see [29]). For any $Q(t) \in[\underline{Q}, \bar{Q}]$, the following inequality holds:

$$
\|Q(t)\| \leq\left\|Q^{*}\right\|+\left\|Q_{*}\right\|,
$$

where $Q^{*}=(\bar{Q}+\underline{Q}) / 2, Q_{*}=(\bar{Q}-\underline{Q}) / 2$. 
Lemma 5 (see [45]). Let scalar $\varepsilon>0, x, y \in R^{n}$, and $A \in$ $R^{n \times n}$, then

$$
x^{T} A y \leq \frac{1}{2 \varepsilon} x^{T} A A^{T} x+\frac{\varepsilon}{2} y^{T} y .
$$

Throughout this paper, the following assumptions are made on (4):

$\left(A_{1}\right) I_{i}(t)$ is an almost periodic function.

$\left(A_{2}\right) g_{i}: R \rightarrow R$ is a nondecreasing continuous function.

$\left(A_{3}\right) \tau_{i}(t)$ is an almost periodic function, and $0<$ $\tau_{i}(t)<\tau, \dot{\tau}_{i}(t) \leq \mu<1, \tau$ and $\mu$ are constants.

\section{Main Results}

In this section, the main results concerned with the existence, uniqueness, and global exponential stability of the almost periodic solution are addressed for the memristive neural network in (4).

Theorem 6. Under the assumptions $\left(A_{1}\right)-\left(A_{3}\right)$, if there exists a diagonal matrix $P=\operatorname{diag}\left(p_{1}, p_{2}, \ldots, p_{n}\right)>0$ such that

$$
\frac{1}{1-\mu} U+\mathscr{P}\left(\left\|B^{*}\right\|+\left\|B_{*}\right\|\right)^{2} U-S<0
$$

where $\mathscr{P}=\max p_{i}, U$ is the identity matrix, $S=\left(s_{i j}\right)_{n \times n}, s_{i i}=$ $-2 p_{i} \bar{a}_{i i}, s_{i j}=-\max \left\{\left|p_{i} \bar{a}_{i j}+p_{j} \bar{a}_{j i}\right|,\left|p_{i} \underline{a}_{i j}+p_{j} \underline{a}_{j i}\right|\right\}, i \neq j, B^{*}=$ $(\bar{B}+\underline{B}) / 2$, and $B_{*}=(\bar{B}-\underline{B}) / 2$. then

(1) For any initial value $x(t)=\varphi(t) \in C\left([-\tau, 0] ; R^{n}\right)$, there exists a solution of the memristive neural network (4) on $[0,+\infty)$, and this solution is asymptotically almost periodic.

(2) The memristive neural network (4) has a unique almost periodic solution which is globally exponentially stable.

Proof. We should prove this theorem in four steps.

Step 1. In this step, we will prove the existence of the solution, that is, prove that the system (4) has a global solution for any initial value $x(t)=\varphi(t) \in C\left([-\tau, 0] ; R^{n}\right)$.

Similar to the proof of Lemma 1 in [37], under the assumptions of Theorem 6 , it is easy to obtain the existence of the local solution of (4) with initial value $x(t)=\varphi(t) \epsilon$ $C\left([-\tau, 0] ; R^{n}\right)$ on $[0, T(\varphi))$, where $T(\varphi) \in(0,+\infty)$ or $T(\varphi)=$ $+\infty$, and $[0, T(\varphi))$ is the maximal right-side existence interval of the local solution.

Due to $\underline{d}_{i}>0, i=1,2, \ldots, n$, by (12) we can choose constants $\delta>0$ and $\nu>0$, such that $0<\delta<\underline{d}_{i}, i=1,2, \ldots, n$, $(\delta+3 \nu) U-2 \underline{D}<0$, and

$$
\frac{e^{\delta t}}{1-\mu} U+\mathscr{P}\left(\left\|B^{*}\right\|+\left\|B_{*}\right\|\right)^{2} U-S<0 .
$$

Without loss of generality, we suppose that $g(0)=0$. Let scalars $\alpha>0, \beta>0$. Consider a Lyapunov functional defined by

$$
V(t)=V_{1}(t)+V_{2}(t)+V_{3}(t), \quad t \geq 0,
$$

where

$$
\begin{gathered}
V_{1}(t)=e^{\delta t} x^{T}(t) x(t), \\
V_{2}=2 \alpha e^{\delta t} \sum_{i=1}^{n} p_{i} \int_{0}^{x_{i}(t)} g_{i}(\theta) d \theta, \\
V_{3}=\frac{\alpha+\beta}{1-\mu} \int_{t-\tau(t)}^{t} g^{T}(x(\theta)) g(x(\theta)) e^{\delta(\theta+\tau)} d \theta .
\end{gathered}
$$

Calculate the time derivative of $V(t)$ along the local solution of $(4)$ on $[0, T(\varphi))$. By (8) and Lemma 5 , we have

$$
\begin{aligned}
\dot{V}_{1}(t)= & \delta e^{\delta t} x^{T}(t) x(t)+2 e^{\delta t} x^{T}(t) \dot{x}(t) \\
= & \delta e^{\delta t} x^{T}(t) x(t)-2 e^{\delta t} x^{T}(t) \mathscr{D}(t) x(t) \\
& +2 e^{\delta t} x^{T}(t) \mathscr{A}(t) g(x(t)) \\
& +2 e^{\delta t} x^{T}(t) \mathscr{B}(t) g(x(t-\tau(t))) \\
& +2 e^{\delta t} x^{T}(t) I(t) \\
\leq & e^{\delta t} x^{T}(t)(\delta U-2 \mathscr{D}(t)) x(t)+v e^{\delta t} x^{T}(t) x(t) \\
& +\frac{e^{\delta t}}{v} g^{T}(x(t)) \mathscr{A}(t)^{T} \mathscr{A}(t) g(x(t)) \\
& +v e^{\delta t} x^{T}(t) x(t) \\
& +\frac{e^{\delta t}}{v} g^{T}(x(t-\tau(t))) \mathscr{B}(t)^{T} \mathscr{B}(t) g(x(t-\tau(t))) \\
& +v e^{\delta t} x^{T}(t) x(t)+\frac{e^{\delta t}}{v} I^{T}(t) I(t) \\
\leq & e^{\delta t} x^{T}(t)((\delta+3 v) U-2 \mathscr{D}(t)) x(t) \\
& +\frac{e^{\delta t}}{v} I^{T}(t) I(t) \\
& +\frac{e^{\delta t}}{v} g^{T}(x(t))\|\mathscr{A}(t)\|^{2} g(x(t)) \\
& +\frac{e^{\delta t}}{v} g^{T}(x(t-\tau(t)))\|\mathscr{B}(t)\|^{2} g(x(t-\tau(t))) \\
& \frac{e^{\delta t}}{v} I^{T}(t) I(t) \\
& g^{T}(x(t-\tau(t)))\|\mathscr{B}(t)\|^{2} g(x(t-\tau(t))) \\
& \\
&
\end{aligned}
$$

By the assumptions $\left(A_{2}\right)$ and $\left(A_{3}\right)$, for any constant $\gamma>0$, we have

$$
\begin{aligned}
\dot{V}_{2}(t)= & 2 \alpha \delta e^{\delta t} \sum_{i=1}^{n} p_{i} \int_{0}^{x_{i}(t)} g_{i}(\theta) d \theta \\
& +2 \alpha e^{\delta t} \sum_{i=1}^{n} p_{i} g_{i}\left(x_{i}(t)\right) \dot{x}_{i}(t)
\end{aligned}
$$




$$
\begin{aligned}
& \leq 2 \alpha \delta e^{\delta t} \sum_{i=1}^{n} p_{i} g_{i}\left(x_{i}(t)\right) x_{i}(t) \\
& +2 \alpha e^{\delta t} \sum_{i=1}^{n} p_{i} g_{i}\left(x_{i}(t)\right) \dot{x}_{i}(t) \\
& =2 \alpha \delta e^{\delta t} g^{T}(x(t)) P x(t) \\
& +2 \alpha e^{\delta t} g^{T}(x(t)) P \dot{x}(t) \\
& =2 \alpha \delta e^{\delta t} g^{T}(x(t)) P x(t) \\
& -2 \alpha e^{\delta t} g^{T}(x(t)) P \mathscr{D}(t) x(t) \\
& +2 \alpha e^{\delta t} g^{T}(x(t)) P \mathscr{A}(t) g(x(t)) \\
& +2 \alpha e^{\delta t} g^{T}(x(t)) P \mathscr{B}(t) g(x(t-\tau(t))) \\
& +2 \alpha e^{\delta t} g^{T}(x(t)) P I(t) \\
& \leq 2 \alpha e^{\delta t} g^{T}(x(t)) P(\delta I-\mathscr{D}(t))(t) x(t) \\
& +\alpha e^{\delta t} g^{T}(x(t))\left(P \mathscr{A}(t)+\mathscr{A}(t)^{T} P\right) g(x(t)) \\
& +\alpha e^{\delta t} g^{T}(x(t)) P \mathscr{B}(t)(P \mathscr{B}(t))^{T} g(x(t)) \\
& +\alpha e^{\delta t} g^{T}(x(t-\tau(t))) g(x(t-\tau(t))) \\
& +\gamma \alpha e^{\delta t} g^{T}(x(t)) g(x(t))+\frac{\alpha \mathscr{P}^{2} e^{\delta t}}{\gamma} I^{T}(t) I(t) \\
& \leq \alpha e^{\delta t} g^{T}(x(t))\left(P \mathscr{A}(t)+\mathscr{A}(t)^{T} P\right. \\
& \left.+P \mathscr{B}(t)(P \mathscr{B}(t))^{T}+\gamma U\right) g(x(t)) \\
& +\alpha e^{\delta t} g^{T}(x(t-\tau(t))) g(x(t-\tau(t))) \\
& +\frac{\alpha \mathscr{P}^{2} e^{\delta t}}{\gamma} I^{T}(t) I(t) . \\
& \dot{V}_{3}=\frac{\alpha+\beta}{1-\mu}\left(e^{\delta(t+\tau)} g^{T}(x(t)) g(x(t))-e^{\delta(t-\tau(t)+\tau)}\right. \\
& \times(1-\dot{\tau}(t)) g^{T}(x(t-\tau(t))) \\
& \times g(x(t-\tau(t)))) \\
& \leq \frac{\alpha+\beta}{1-\mu} g^{T}(x(t)) g(x(t)) e^{\delta(t+\tau)} \\
& -(\alpha+\beta) g^{T}(x(t-\tau(t))) g(x(t-\tau(t))) e^{\delta t} .
\end{aligned}
$$

From (16) and (17) and by Lemma 4, one yields

$$
\begin{aligned}
\dot{V}(t) \leq & \alpha e^{\delta t} g^{T}(x(t)) \\
& \times\left(P \mathscr{A}(t)+\mathscr{A}(t)^{T} P+P B(t)(P B(t))^{T}\right.
\end{aligned}
$$

$$
\begin{gathered}
+\left(\gamma+\frac{\|\mathscr{A}(t)\|^{2}}{\alpha \nu}+\frac{e^{\delta \tau}}{1-\mu}\right. \\
\left.\left.\times\left(1+\frac{\beta}{\alpha}\right)\right) U\right) g(x(t)) \\
+e^{\delta t} g^{T}(x(t-\tau(t)))\left(\frac{\|\mathscr{B}(t)\|^{2}}{\nu}-\beta\right) \\
\times g(x(t-\tau(t)))+e^{\delta t}\left(\frac{1}{\nu}+\frac{\alpha \mathscr{P}^{2}}{\gamma}\right)\|I(t)\|^{2} \\
\leq \alpha e^{\delta t}\left|g^{T}(x(t))\right| \\
\times\left(\mathscr{P}^{2}\left(\left\|B^{*}\right\|+\left\|B_{*}\right\|\right)^{2} U-S\right. \\
+\left(\gamma+\frac{\left(\left\|A_{*}\right\|+\left\|A^{*}\right\|\right)^{2}}{\alpha \nu}\right. \\
+e^{\delta t}\left(\frac{1}{v}+\frac{\alpha \mathscr{P}^{2}}{\gamma}\right)\|I(t)\|^{2}, \\
\times\left(\frac{\left(\left\|B_{*}\right\|+\left\|B^{*}\right\|^{2}\right.}{v t}-\beta\right) g(x(t-\tau(t))) \\
g^{T}(x(t-\tau(t))) \\
\left.\left.\left.+\frac{\beta}{\alpha}\right)\right) U\right)|g(x(t))|
\end{gathered}
$$

where $A^{*}=(\bar{A}+\underline{A}) / 2, A_{*}=(\bar{A}-\underline{A}) / 2$. By (13), we can choose suitable constants $\gamma>0, \alpha>0, \nu>0$, and $\beta>0$, such that

$$
\begin{gathered}
\frac{\left(\left\|B_{*}\right\|+\left\|B^{*}\right\|\right)^{2}}{\nu}-\beta<0, \\
\mathscr{P}^{2}\left(\left\|B^{*}\right\|+\left\|B_{*}\right\|\right)^{2} U-S \\
+\left(\gamma+\frac{\left(\left\|A_{*}\right\|+\left\|A^{*}\right\|\right)^{2}}{\alpha \nu}+\frac{e^{\delta \tau}}{1-\mu}\left(1+\frac{\beta}{\alpha}\right)\right) U<0 .
\end{gathered}
$$

This implies that

$$
\dot{V}(t) \leq e^{\delta t}\left(\frac{1}{v}+\frac{\alpha \mathscr{P}^{2}}{\gamma}\right)\|I(t)\|^{2} .
$$

Moreover, by the assumption $\left(A_{1}\right)$, we can obtain that $I(t)$ is a bounded function. Hence, there exist a constant $M>0$, such that

$$
0<\left(\frac{1}{\nu}+\frac{\alpha \mathscr{P}^{2}}{\gamma}\right)\|I(t)\|^{2}<M, \quad t \geq 0 .
$$

By (20) and (21), it follows that

$$
\dot{V}(t) \leq M e^{\delta t}, \quad t \in[0, T(\varphi)) .
$$


From the definition of $V(t)$ and (22), we have

$$
\begin{aligned}
e^{\delta t}\|x(t)\|^{2} & \leq V(t) \leq V(0)+\int_{0}^{t} \dot{V}(s) d s \\
& \leq V(0)+\int_{0}^{t} M e^{\delta s} d s \\
& =V(0)+\frac{M}{\delta}\left(e^{\delta t}-1\right), \quad t \in[0, T(\varphi)) .
\end{aligned}
$$

Thus,

$$
\begin{aligned}
\|x(t)\|^{2} & \leq e^{-\delta t} V(0)+\frac{M}{\delta}\left(1-e^{-\delta t}\right) \\
& \leq V(0)+\frac{M}{\delta}, \quad t \in[0, T(\varphi)) .
\end{aligned}
$$

This shows that the local solution $x(t)$ of (4) is bounded on $[0, T(\varphi))$ and hence is defined on $[0,+\infty)$. That is, the system (4) has a global solution for any initial value $x(t)=\varphi(t) \epsilon$ $C\left([-\tau, 0] ; R^{n}\right)$.

Step 2. In this step, the global solution $x(t)$ of the system (4) will be proved to be asymptotically almost periodic.

Let $y(t)=x(t+\omega)-x(t)$, then

$$
\begin{aligned}
\dot{y}(t)= & -\mathscr{D}(t) y(t)+\mathscr{A}(t) \tilde{g}(y(t)) \\
& +\mathscr{B}(t) \tilde{g}(y(t-\tau(t)))+\widetilde{I}(\omega, t),
\end{aligned}
$$

where

$$
\begin{gathered}
\tilde{g}(y(t))=g(x(t+\omega))-g(x(t)), \\
\tilde{g}(y(t-\tau(t)))=g(x(t+\omega-\tau(t)))-g(x(t-\tau(t))), \\
\tilde{I}(\omega, t)=I(t+\omega)-I(t) .
\end{gathered}
$$

Similar to $V(t)$, define Lyapunov functional $W(t)$ as

$$
\begin{aligned}
W(t)= & e^{\delta t} y^{T}(t) y(t)+2 \alpha e^{\delta t} \sum_{i=1}^{n} p_{i} \int_{0}^{y_{i}(t)} \tilde{g}_{i}(\theta) d \theta \\
& +\frac{\alpha+\beta}{1-\mu} \int_{t-\tau(t)}^{t} \tilde{g}^{T}(y(\theta)) \tilde{g}(y(\theta)) e^{\delta(\theta+\tau)} d \theta .
\end{aligned}
$$

Calculate the derivative of $W(t)$ along the solution of the system (25). Arguing as in Step 1, we can choose the appropriate positive constants $\nu, \gamma, \alpha$, and $\delta$, such that

$$
\dot{W}(t) \leq e^{\delta t}\left(\frac{1}{\nu}+\frac{\alpha \mathscr{P}^{2}}{\gamma}\right)\|\tilde{I}(\omega, t)\|^{2}, \quad t>0 .
$$

By the assumption $\left(A_{1}\right), I_{i}(t)$ is an almost periodic function. Thus, by Definition 1 , for any $\varepsilon>0$, there exists $l=l(\varepsilon)>0$, and for any interval with length $l$, there exists a scalar $\omega$ in this interval, such that

$$
\left(\frac{1}{\nu}+\frac{\alpha \mathscr{P}^{2}}{\gamma}\right)\|\tilde{I}(\omega, t)\|^{2} \leq \frac{1}{2} \varepsilon^{2} \delta, \quad t>0 .
$$

It follows from (28) and (29) that $\dot{W}(t) \leq(1 / 2) \varepsilon^{2} \delta e^{\delta t}, t>0$, which implies

$$
\begin{aligned}
e^{\delta t}\|y(t)\|^{2} & \leq W(t) \leq W(0)+\int_{0}^{t} \frac{1}{2} \delta \varepsilon^{2} e^{\delta s} d s \\
& =W(0)+\frac{1}{2} \varepsilon^{2}\left(e^{\delta t}-1\right), \quad t>0 \\
& \|x(t+\omega)-x(t)\| \\
& \leq\left(e^{-\delta t} W(0)+\frac{1}{2} \varepsilon^{2}\left(1-e^{-\delta t}\right)\right)^{1 / 2}, \quad t>0
\end{aligned}
$$

Therefore, there exists a constant $T>0$; when $t>T$, we have

$$
\|x(t+\omega)-x(t)\| \leq \varepsilon .
$$

This shows that the solution of the system (4) is asymptotically almost periodic.

Step 3. In this step, we will prove that the system (4) has an almost periodic solution.

Let $x(t)$ be the solution of the system (4) with the initial value $x(t)=\varphi(t) \in C\left([-\tau, 0] ; R^{n}\right)$, then $x(t)$ satisfies (8). Take a sequence $\left\{t_{k}\right\}, t_{1}<t_{2}<\cdots<t_{k}<t_{k+1}<\cdots, \lim _{k \rightarrow+\infty} t_{k}=$ $+\infty$. It is easy to derive that the function sequence $\left\{x\left(t+t_{k}\right)\right\}$ is equicontinuous and uniformly bounded. Hence, by using Arzela-Ascoli theorem and diagonal selection principle, a subsequence of $\left\{t_{k}\right\}$ (still denoted by $\left\{t_{k}\right\}$ ) can be selected, such that $\left\{x\left(t+t_{k}\right)\right\}$ uniformly converges to a continuous function $x^{*}(t)$ on any compact set of $R$.

By applying Lebesgue's dominated convergence theorem on (8), we can obtain that

$$
\begin{aligned}
x^{*}( & +h)-x^{*}(t) \\
= & \lim _{k \rightarrow+\infty}\left(x\left(t+t_{k}+h\right)-x\left(t+t_{k}\right)\right) \\
= & \lim _{k \rightarrow+\infty} \int_{t}^{t+h}\left(-\mathscr{D}(t) x\left(t_{k}+\theta\right)\right. \\
& +\mathscr{A}(t) g\left(x\left(t_{k}+\theta\right)\right) \\
& \left.+\mathscr{B}(t) g\left(x\left(t_{k}+\theta-\tau\left(t_{k}+\theta\right)\right)\right)+I\left(t_{k}+\theta\right)\right) d \theta \\
= & \int_{t}^{t+h}\left(-\mathscr{D}(t) x^{*}(\theta)+\mathscr{A}(t) g\left(x^{*}(\theta)\right)\right. \\
& \left.+\mathscr{B}(t) g\left(x^{*}(\theta-\tau(\theta))\right)+I(\theta)\right) d \theta
\end{aligned}
$$

for any $t \in[-\tau,+\infty)$ and $h \in R$. This implies that $x^{*}(t)$ is a solution of (4). By the result obtained in Step 2, $x(t)$ is asymptotically almost periodic. That is, for any $\varepsilon>0$, there exist $T>0, l=l(\varepsilon)$, and for any interval with length $l$, there exists a scalar $\omega$ in this interval, such that $\|x(t+\omega)-x(t)\| \leq \varepsilon$ for all $t \geq T$. Thus, there exists a constant $K>0$; for all $t \in[-\tau,+\infty)$ and $k>K$, we can get that

$$
\left\|x\left(t+t_{k}+\omega\right)-x\left(t+t_{k}\right)\right\| \leq \varepsilon .
$$


Let $k \rightarrow+\infty$ in (33), it follows that $\left\|x^{*}(t+\omega)-x^{*}(t)\right\| \leq \varepsilon$ for all $t \in[-\tau,+\infty)$. This shows that $x^{*}(t)$ is the almost periodic solution of (4). The proof of the existence of the almost periodic solution has been completed.

Step 4. In this step, we will prove that the uniqueness and global exponential stability of the almost periodic solution for the system (4).

Let $x(t)$ be any solution of (4), and let $x^{*}(t)$ be an almost periodic solution of (4). Set $z(t)=x(t)-x^{*}(t)$, then

$$
\begin{aligned}
\dot{z}(t)= & -\mathscr{D}(t) z(t)+\mathscr{A}(t) \hat{g}(z(t)) \\
& +\mathscr{B}(t) \hat{g}(z(t-\tau(t))),
\end{aligned}
$$

where

$$
\begin{gathered}
\widehat{g}(z(t))=g(x(t))-g\left(x^{*}(t)\right), \\
\widehat{g}(z(t-\tau(t)))=g(x(t-\tau(t)))-g\left(x^{*}(t-\tau(t))\right) .
\end{gathered}
$$

Similar to $V(t)$, define Lyapunov functional $L(t)$ as

$$
L(t)=L_{1}(t)+L_{2}(t)+L_{3}(t),
$$

where

$$
\begin{gathered}
L_{1}(t)=e^{\delta t} z^{T}(t) z(t), \\
L_{2}(t)=2 \alpha e^{\delta t} \sum_{i=1}^{n} p_{i} \int_{0}^{z_{i}(t)} \widehat{g}_{i}(\theta) d \theta, \\
L_{3}(t)=(\alpha+\beta) \int_{t-\tau(t)}^{t} \hat{g}^{T}(z(\theta)) \hat{g}(z(\theta)) e^{\delta(\theta+\tau)} d \theta .
\end{gathered}
$$

Arguing as in Step 1, we have

$$
\begin{aligned}
\dot{L}_{1}(t) \leq & \frac{e^{\delta t}}{\nu} \widehat{g}^{T}(z(t))\|\mathscr{A}(t)\|^{2} \widehat{g}(z(t)) \\
& +\frac{e^{\delta t}}{\nu} \widehat{g}^{T}(z(t-\tau(t)))\|\mathscr{B}(t)\|^{2} \widehat{g}(z(t-\tau(t))), \\
\dot{L}_{2}(t) \leq & \alpha e^{\delta t} \widehat{g}(z(t))\left(P \mathscr{A}(t)+\mathscr{A}(t)^{T} P\right. \\
& \left.+P \mathscr{B}(t)(P \mathscr{B}(t))^{T}\right) \hat{g}(z(t)) \\
& +\alpha e^{\delta t} \widehat{g}^{T}(z(t-\tau(t))) \hat{g}(z(t-\tau(t))), \\
\dot{L}_{3}(t) \leq & \frac{\alpha+\beta}{1-\mu} \hat{g}(z(t)) \hat{g}(z(t)) e^{\delta(t+\tau)} \\
& -(\alpha+\beta) \hat{g}^{T}\left(z(t-\tau(t)) \hat{g}(z(t-\tau(t))) e^{\delta t} .\right.
\end{aligned}
$$

Thus

$$
\begin{array}{r}
\dot{L}(t) \leq \alpha e^{\delta t} \widehat{g}^{T}(z(t))\left(P \mathscr{A}(t)+\mathscr{A}(t) P+P \mathscr{B}(t)(P \mathscr{B}(t))^{T}\right. \\
\left.+\left(\frac{\|\mathscr{A}(t)\|^{2}}{\alpha \nu}+\frac{e^{\delta \tau}}{1-\mu}\left(1+\frac{\beta}{\alpha}\right)\right) U\right)
\end{array}
$$

$$
\begin{aligned}
& \times \hat{g}(z(t)) \\
& +e^{\delta t} \hat{g}(z(t-\tau(t)))\left(\frac{\|\mathscr{B}(t)\|^{2}}{\nu}-\beta\right) \widehat{g}(z(t-\tau(t))) \\
& \leq \alpha e^{\delta t}\left|g^{T}(x(t))\right| \\
& \times\left(\mathscr{P}^{2}\left(\left\|B^{*}\right\|+\left\|B_{*}\right\|\right)^{2} U-S\right. \\
& \left.\quad+\left(\frac{\left(\left\|A_{*}\right\|+\left\|A^{*}\right\|\right)^{2}}{\alpha \nu}+\frac{e^{\delta \tau}}{1-\mu}\left(1+\frac{\beta}{\alpha}\right)\right) U\right) \\
& \times|g(x(t))| \\
& +e^{\delta t} g^{T}(x(t-\tau(t)))\left(\frac{\left(\left\|B_{*}\right\|+\left\|B^{*}\right\|\right)^{2}}{\nu}-\beta\right) \\
& \times g(x(t-\tau(t))) .
\end{aligned}
$$

By (13), we can choose appropriate constants $\gamma>0, \alpha>0$, $\nu>0$, and $\beta>0$, such that

$$
\begin{gathered}
\mathscr{P}^{2}\left(\left\|B^{*}\right\|+\left\|B_{*}\right\|\right)^{2} U-S \\
+\left(\frac{\left(\left\|A_{*}\right\|+\left\|A^{*}\right\|\right)^{2}}{\alpha \nu}+\frac{e^{\delta \tau}}{1-\mu}\left(1+\frac{\beta}{\alpha}\right)\right) U<0, \\
\frac{\left(\left\|B_{*}\right\|+\left\|B^{*}\right\|\right)^{2}}{\nu}-\beta<0 .
\end{gathered}
$$

This implies that $\dot{L}(t) \leq 0$. Therefore, combined with the definition of $L(t)$, it follows that

$$
\|z(t)\|^{2}=\left\|x(t)-x^{*}(t)\right\|^{2} \leq e^{-\delta t} L(t) \leq L(0) e^{-\delta t} .
$$

This shows that the almost periodic solution $x^{*}(t)$ of the system (4) is globally exponentially stable. Consequently, the periodic solution $x^{*}(t)$ is unique. This completes the proof of Theorem 6 .

Notice that periodic function can be regarded as special almost periodic function. Hence, when $I_{i}(t)$ is a periodic external input in the system (4), we can get the following corollary.

Corollary 7. Suppose that the assumptions $\left(A_{2}\right)-\left(A_{3}\right)$ hold. If there exists a diagonal matrix $P=\operatorname{diag}\left(p_{1}, p_{2}, \ldots, p_{n}\right)>0$ such that

$$
\frac{1}{1-\mu} U+\mathscr{P}\left(\left\|B^{*}\right\|+\left\|B_{*}\right\|\right)^{2} U-S<0,
$$

where $\mathscr{P}=\max p_{i}, U$ is the identity matrix, and $S=$ $\left(s_{i j}\right)_{n \times n}, s_{i i}=-2 p_{i} \bar{a}_{i i}, s_{i j}=-\max \left\{\left|p_{i} \bar{a}_{i j}+p_{j} \bar{a}_{j i}\right|, \mid p_{i} \underline{a}_{i j}+\right.$ $\left.p_{j} \underline{a}_{j i} \mid\right\}$, and $i \neq j$, then one has the following.

(1) For any initial value $x(t)=\varphi(t) \in C\left([-\tau, 0] ; R^{n}\right)$, there exists a solution of the memristive neural network (4) on $[0,+\infty)$, and this solution is asymptotically periodic. 
(2) The memristive neural network (4) has a unique periodic solution which is globally exponentially stable.

When $I_{i}(t)$ is a constant external input $I_{i}$, the system (4) changes as

$$
\begin{aligned}
\dot{x}_{i}(t)= & -d_{i}\left(x_{i}(t)\right) x_{i}(t)+\sum_{j=1}^{n} a_{i j}\left(x_{i}(t)\right) g_{j}\left(x_{j}(t)\right) \\
& +\sum_{j=1}^{n} b_{i j}\left(x_{i}(t)\right) g_{j}\left(x_{j}(t-\tau(t))\right)+I_{i}, \quad i=1,2, \ldots, n .
\end{aligned}
$$

Since a constant can be also regarded as a special almost periodic function, by applying Theorem 6 on the neural network (43), we have the following.

Corollary 8. Suppose that the assumptions $\left(A_{2}\right)-\left(A_{3}\right)$ hold. If there exists a diagonal matrix $P=\operatorname{diag}\left(p_{1}, p_{2}, \ldots, p_{n}\right)>0$ such that

$$
\frac{1}{1-\mu} U+\mathscr{P}\left(\left\|B^{*}\right\|+\left\|B_{*}\right\|\right)^{2} U-S<0
$$

where $\mathscr{P}=\max p_{i}, U$ is the identity matrix, and $S=$ $\left(s_{i j}\right)_{n \times n}, s_{i i}=-2 p_{i} \bar{a}_{i i}, s_{i j}=-\max \left\{\left|p_{i} \bar{a}_{i j}+p_{j} \bar{a}_{j i}\right|, \mid p_{i} \underline{a}_{i j}+\right.$ $\left.p_{j} \underline{a}_{j i} \mid\right\}$, and $i \neq j$, then one has the following.

(1) For any initial value $x(t)=\varphi(t) \in C\left([-\tau, 0] ; R^{n}\right)$, there exists a solution of the memristive neural network (43) on $[0,+\infty)$.

(2) The memristive neural network (43) has a unique equilibrium point which is globally exponentially stable.

\section{Illustrative Examples}

In this section, two examples will be given to illustrate the effectiveness of the results obtained in this paper.

Example 1. Consider the second-order memristive neural network with time-varying delays in (4) described by

$$
\begin{aligned}
\left(\begin{array}{c}
\dot{x}_{1}(t) \\
\dot{x}_{2}(t)
\end{array}\right)= & \left(\begin{array}{cc}
d_{11}\left(x_{1}\right) & 0 \\
0 & d_{22}\left(x_{2}\right)
\end{array}\right)\left(\begin{array}{l}
x_{1}(t) \\
x_{2}(t)
\end{array}\right) \\
& +\left(\begin{array}{cc}
a_{11}\left(x_{1}\right) & a_{12}\left(x_{1}\right) \\
a_{21}\left(x_{2}\right) & a_{22}\left(x_{2}\right)
\end{array}\right)\left(\begin{array}{l}
x_{1}^{3}(t) \\
x_{2}^{3}(t)
\end{array}\right) \\
& +\left(\begin{array}{ll}
b_{11}\left(x_{1}\right) & b_{12}\left(x_{1}\right) \\
b_{21}\left(x_{2}\right) & b_{22}\left(x_{2}\right)
\end{array}\right)\left(\begin{array}{l}
x_{1}^{3}(t-0.5(\sin t+1)) \\
x_{2}^{3}(t-0.5(\sin t+1))
\end{array}\right) \\
& +\left(\begin{array}{c}
2-4 \sin t \\
-3-4 \cos \sqrt{2} t
\end{array}\right),
\end{aligned}
$$

where

$$
\begin{gathered}
d_{11}\left(x_{1}\right)= \begin{cases}0.8, & \left|x_{1}(t)\right|<1.5, \\
1, & \left|x_{1}(t)\right|>1.5,\end{cases} \\
d_{22}\left(x_{2}\right)= \begin{cases}2, & \left|x_{2}(t)\right|<1.5, \\
2.4, & \left|x_{2}(t)\right|>1.5,\end{cases} \\
a_{11}\left(x_{1}\right)= \begin{cases}-150, & \left|x_{1}(t)\right|<1.5, \\
-145, & \left|x_{1}(t)\right|>1.5,\end{cases} \\
a_{12}\left(x_{1}\right)= \begin{cases}0, & \left|x_{1}(t)\right|<1.5, \\
1, & \left|x_{1}(t)\right|>1.5,\end{cases} \\
a_{21}\left(x_{2}\right)= \begin{cases}0, & \left|x_{2}(t)\right|<1.5, \\
2, & \left|x_{2}(t)\right|>1.5,\end{cases} \\
a_{22}\left(x_{2}\right)= \begin{cases}-162, & \left|x_{2}(t)\right|<1.5, \\
-160, & \left|x_{2}(t)\right|>1.5,\end{cases} \\
b_{21}\left(x_{2}\right)= \begin{cases}1, & \left|x_{2}(t)\right|<1.5, \\
2, & \left|x_{2}(t)\right|>1.5,\end{cases} \\
b_{11}\left(x_{1}\right)= \begin{cases}-1, & \left|x_{1}(t)\right|<1.5, \\
3, & \left|x_{1}(t)\right|>1.5,\end{cases} \\
\left.b_{1}\right)= \begin{cases}2, & \left|x_{1}(t)\right|<1.5, \\
4, & \left|x_{1}(t)\right|>1.5,\end{cases}
\end{gathered}
$$

It is obvious that $\tau=1, \mu=0.5$. Choose the positive definite diagonal matrix $P=\operatorname{diag}(1,0.1)$, then $S=\left(\begin{array}{cc}290.0 & -1.2 \\ -1.2 & 320.0\end{array}\right)$. It is easy to check that

$$
\begin{aligned}
& \frac{1}{1-\mu} U+\mathscr{P}\left(\left\|B^{*}\right\|+\left\|B_{*}\right\|\right)^{2} U-S \\
& \quad=\left(\begin{array}{cc}
-226.7157 & 1.2000 \\
1.2000 & -256.7157
\end{array}\right)<0 .
\end{aligned}
$$

All conditions of Theorem 6 hold; hence the memristive neural network in this example has a unique almost periodic solution which is globally exponentially stable.

Figure 1 displays the state trajectory of the network with initial condition $\left(\varphi_{1}(t), \varphi_{2}(t)\right)^{T}=\left(-0.5 t^{2}, 0.5 t\right)^{T}, t \in[-1,0)$. It can be seen that this trajectory converges to the unique almost periodic solution of the network. This is in accordance with the conclusion of Theorem 6 .

Example 2. Consider the third-order memristive neural network with time-varying delays in (4) described by

$$
\left(\begin{array}{l}
\dot{x}_{1}(t) \\
\dot{x}_{2}(t) \\
\dot{x}_{3}(t)
\end{array}\right)=\left(\begin{array}{ccc}
d_{11}\left(x_{1}\right) & 0 & 0 \\
0 & d_{22}\left(x_{2}\right) & 0 \\
0 & 0 & d_{33}\left(x_{3}\right)
\end{array}\right)\left(\begin{array}{l}
x_{1}(t) \\
x_{2}(t) \\
x_{3}(t)
\end{array}\right)
$$


Journal of Applied Mathematics

9

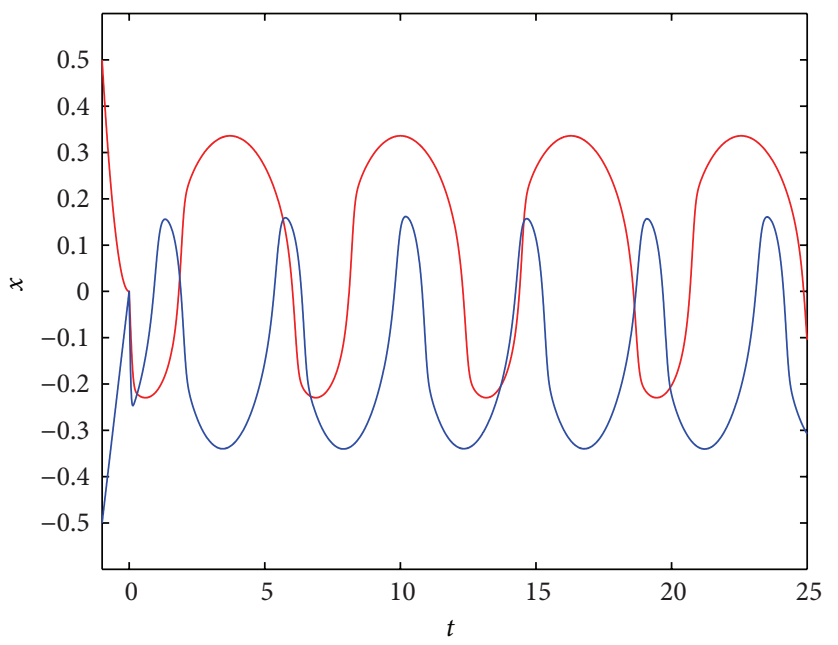

$-x_{1}$

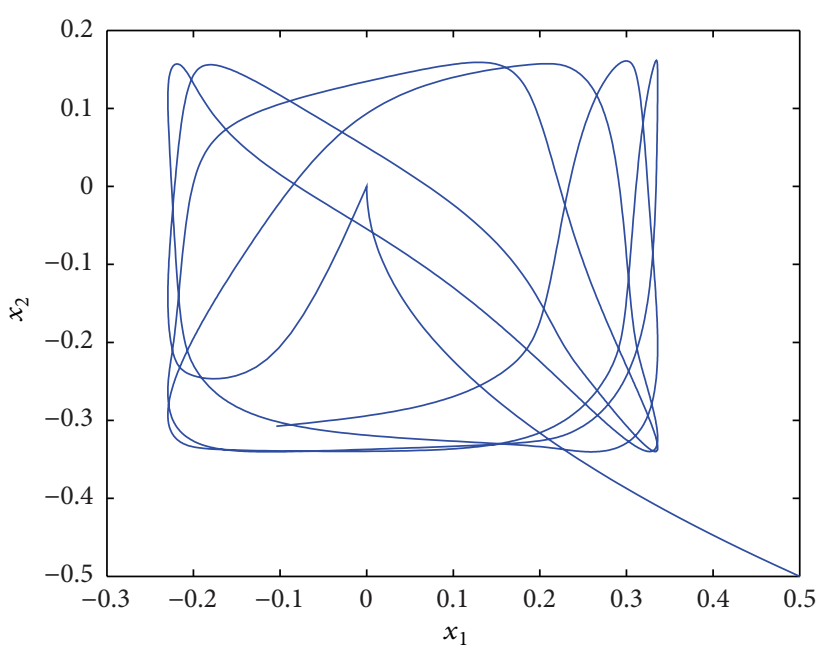

(b)

FIGURE 1: The state trajectory of the network with the initial condition $\left(\varphi_{1}(t), \varphi_{2}(t)\right)^{T}=\left(-0.5 t^{2}, 0.5 t\right)^{T}, t \in[-1,0)$.

$$
\begin{aligned}
& +\left(\begin{array}{lll}
a_{11}\left(x_{1}\right) & a_{12}\left(x_{1}\right) & a_{13}\left(x_{1}\right) \\
a_{21}\left(x_{2}\right) & a_{22}\left(x_{2}\right) & a_{23}\left(x_{2}\right) \\
a_{31}\left(x_{3}\right) & a_{32}\left(x_{3}\right) & a_{33}\left(x_{3}\right)
\end{array}\right)\left(\begin{array}{l}
x_{1}^{3}(t) \\
x_{2}^{3}(t) \\
x_{3}^{3}(t)
\end{array}\right) \\
& +\left(\begin{array}{llll}
b_{11}\left(x_{1}\right) & b_{12}\left(x_{1}\right) & b_{13}\left(x_{1}\right) \\
b_{21}\left(x_{2}\right) & b_{22}\left(x_{2}\right) & b_{23}\left(x_{2}\right) \\
b_{31}\left(x_{3}\right) & b_{32}\left(x_{3}\right) & b_{33}\left(x_{3}\right)
\end{array}\right) \\
& \times\left(\begin{array}{c}
x_{1}^{3}(t-0.5(\cos t+1)) \\
x_{2}^{3}(t-0.5(\cos t+1)) \\
x_{3}^{3}(t-0.5(\cos t+1))
\end{array}\right) \\
& +\left(\begin{array}{c}
2+2 \sin \sqrt{2} t \\
-3-3 \cos \sqrt{3} t \\
\sin \sqrt{6} t
\end{array}\right)
\end{aligned}
$$

where

$$
\begin{aligned}
& d_{11}\left(x_{1}\right)= \begin{cases}0.1, & \left|x_{1}(t)\right|<1, \\
0.3, & \left|x_{1}(t)\right|>1,\end{cases} \\
& d_{22}\left(x_{2}\right)= \begin{cases}1, & \left|x_{2}(t)\right|<1, \\
1.9, & \left|x_{2}(t)\right|>1,\end{cases} \\
& d_{33}\left(x_{3}\right)= \begin{cases}3, & \left|x_{3}(t)\right|<1, \\
3.3, & \left|x_{3}(t)\right|>1,\end{cases} \\
& a_{11}\left(x_{1}\right)= \begin{cases}-50, & \left|x_{1}(t)\right|<1, \\
-49.3, & \left|x_{1}(t)\right|>1,\end{cases} \\
& a_{12}\left(x_{1}\right)= \begin{cases}-8, & \left|x_{1}(t)\right|<1, \\
-7.6, & \left|x_{1}(t)\right|>1,\end{cases} \\
& a_{13}\left(x_{1}\right)= \begin{cases}-16, & \left|x_{1}(t)\right|<1, \\
-15.4, & \left|x_{1}(t)\right|>1,\end{cases}
\end{aligned}
$$

$$
\begin{aligned}
& a_{21}\left(x_{2}\right)= \begin{cases}-1, & \left|x_{2}(t)\right|<1, \\
-0.7, & \left|x_{2}(t)\right|>1,\end{cases} \\
& a_{22}\left(x_{2}\right)= \begin{cases}-160, & \left|x_{2}(t)\right|<1, \\
-159.5, & \left|x_{2}(t)\right|>1,\end{cases} \\
& a_{23}\left(x_{2}\right)= \begin{cases}2, & \left|x_{2}(t)\right|<1, \\
2.3, & \left|x_{2}(t)\right|>1,\end{cases} \\
& a_{31}\left(x_{3}\right)= \begin{cases}-3, & \left|x_{3}(t)\right|<1, \\
-2.4, & \left|x_{3}(t)\right|>1,\end{cases} \\
& a_{32}\left(x_{3}\right)= \begin{cases}-4, & \left|x_{3}(t)\right|<1, \\
-3.6, & \left|x_{3}(t)\right|>1,\end{cases}
\end{aligned}
$$

$$
\begin{aligned}
a_{33}\left(x_{3}\right) & = \begin{cases}-120, & \left|x_{3}(t)\right|<1, \\
-119.7, & \left|x_{3}(t)\right|>1,\end{cases} \\
b_{11}\left(x_{1}\right) & = \begin{cases}-1, & \left|x_{1}(t)\right|<1, \\
-0.8, & \left|x_{1}(t)\right|>1,\end{cases} \\
b_{12}\left(x_{1}\right) & = \begin{cases}0, & \left|x_{1}(t)\right|<1, \\
0.1, & \left|x_{1}(t)\right|>1,\end{cases} \\
b_{13}\left(x_{1}\right) & = \begin{cases}1, & \left|x_{1}(t)\right|<1, \\
1.2, & \left|x_{1}(t)\right|>1,\end{cases} \\
b_{21}\left(x_{2}\right) & = \begin{cases}-0.5, & \left|x_{2}(t)\right|<1, \\
-0.4, & \left|x_{2}(t)\right|>1,\end{cases} \\
b_{22}\left(x_{2}\right) & = \begin{cases}-0.3, & \left|x_{2}(t)\right|<1, \\
-0.2, & \left|x_{2}(t)\right|>1,\end{cases}
\end{aligned}
$$




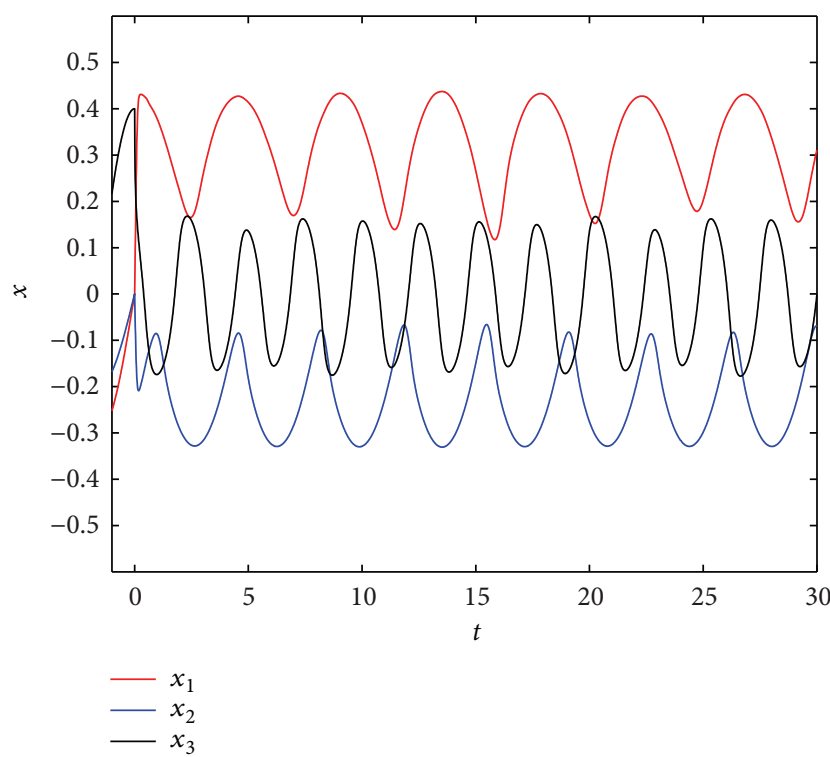

(a)

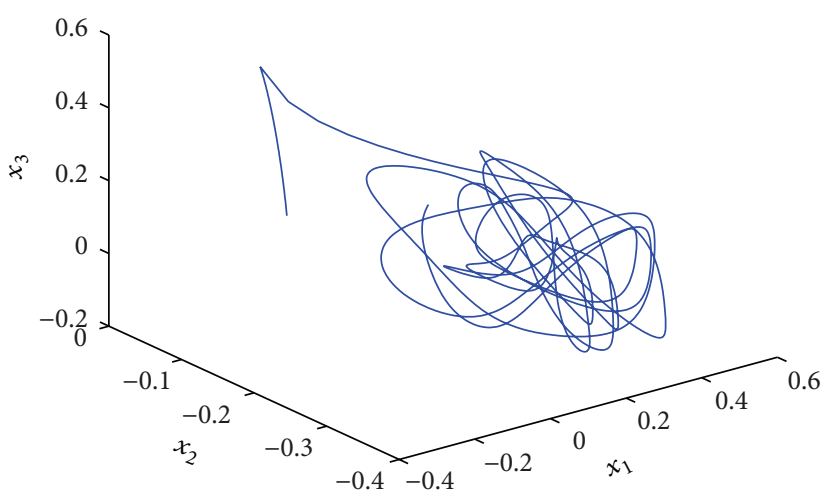

(b)

FIgURE 2: The state trajectory of the network with the initial condition $\left(\varphi_{1}(t), \varphi_{2}(t), \varphi_{3}(t)\right)^{T}=(0.3 \sin t, 0.2 \sin t, 0.4 \cos t)^{T}, t \in[-1,0)$.

$$
\begin{aligned}
& b_{23}\left(x_{2}\right)= \begin{cases}0.7, & \left|x_{2}(t)\right|<1, \\
0.8, & \left|x_{2}(t)\right|>1,\end{cases} \\
& b_{31}\left(x_{3}\right)= \begin{cases}0, & \left|x_{3}(t)\right|<1, \\
0.1, & \left|x_{3}(t)\right|>1,\end{cases} \\
& b_{32}\left(x_{3}\right)= \begin{cases}0, & \left|x_{3}(t)\right|<1, \\
0.2, & \left|x_{3}(t)\right|>1,\end{cases} \\
& b_{33}\left(x_{3}\right)= \begin{cases}-1, & \left|x_{3}(t)\right|<1, \\
-0.6, & \left|x_{3}(t)\right|>1 .\end{cases}
\end{aligned}
$$

It is obvious that $\tau=1, \mu=0.5$. Choose the positive definite diagonal matrix $P=\operatorname{diag}(1,1,0.1)$, then $S=$ $\left(\begin{array}{rrr}98.60 & -9.00 & -16.30 \\ -9.00 & 319.00 & -1.94 \\ -16.30 & -1.94 & 239.40\end{array}\right)$. It is easy to check that

$$
\begin{aligned}
\frac{1}{1-\mu} U+\mathscr{P}\left(\left\|B^{*}\right\|+\left\|B_{*}\right\|\right)^{2} U-S \\
\quad=\left(\begin{array}{ccc}
-91.7253 & 9.0000 & 16.3000 \\
9.0000 & -312.1253 & 1.9400 \\
16.3000 & 1.9400 & -232.5253
\end{array}\right)<0 .
\end{aligned}
$$

All conditions of Theorem 6 hold; hence the memristive neural network in this example has a unique almost periodic solution which is globally exponentially stable.

Figure 2 displays the state trajectory of the network with initial condition $\left(\varphi_{1}(t), \varphi_{2}(t), \varphi_{3}(t)\right)^{T}=(0.3 \sin t, 0.2 \sin t$, $0.4 \cos t)^{T}, t \in[-1,0)$. It can be seen that this trajectory converges to the unique almost periodic solution of the network. This is in accordance with the conclusion of Theorem 6.

\section{Conclusion}

In this paper, the exponential stability issue of the almost periodic solution for memristive neural networks with time-varying delays has been investigated. A sufficient condition has been obtained to ensure the existence, uniqueness, and global exponential stability of the almost periodic solution. As special cases, when the external input is a periodic or constant function in the network, the conditions which ensure the global exponential stability of a unique periodic solution or equilibrium point have been established for the considered memristive neural networks with time-varying delay. Two illustrative examples have been also given to demonstrate the effectiveness and validity of the proposed results in this paper.

In [56], the distributed filtering issue have been studied for a class of time-varying systems over sensor networks with quantization errors and successive packet dropouts. In [57], authors considered the the exponential stabilization of a class of stochastic system with Markovian jump parameters and mode-dependent mixed time delays. In [58], authors discussed the fuzzy-model-based robust fault detection with stochastic mixed time delays and successive packet dropouts. However, the issues of distributed filtering, stochastic stabilization, and robust fault detection have not been investigated for memristive neural networks in the existing literature. These will be the topic of our research on memristive neural networks with mode-dependent mixed time delays and Markovian jump parameters in future.

\section{Acknowledgments}

This work was supported by the Natural Science Foundation of Hebei Province of China (A2011203103) and the Hebei Province Education Foundation of China (2009157). 


\section{References}

[1] L. O. Chua, "Memristor: the missing circuit element," IEEE Transactions on Circuit Theory, vol. 18, no. 5, pp. 507-519, 1971.

[2] D. B. Strukov, G. S. Snider, D. R. Stewart, and R. S. Williams, "The missing memristor found," Nature, vol. 453, pp. 80-83, 2008.

[3] J. M. Tour and T. He, "Electronics: the fourth element," Nature, vol. 453, no. 7191, pp. 42-43, 2008.

[4] R. S. Williams, "How we found the missing Memristor," IEEE Spectrum, vol. 45, no. 12, pp. 28-35, 2008.

[5] M. Di Ventra, Y. V. Pershin, and L. O. Chua, "Circuit elements with memory: memristors, memcapacitors, and meminductors," Proceedings of the IEEE, vol. 97, no. 10, pp. 1717-1724, 2009.

[6] M. Itoh and L. O. Chua, "Memristor oscillators," International Journal of Bifurcation and Chaos, vol. 18, no. 11, pp. 3183-3206, 2008.

[7] Y. V. Pershin and M. Di Ventra, "Spin memristive systems: spin memory effects in semiconductor spintronics," Physical Review $B$, vol. 78, no. 11, Article ID 113309, 4 pages, 2008.

[8] X. Wang, Y. Chen, Y. Gu, and H. Li, "Spintronic memristor temperature sensor," IEEE Electron Device Letters, vol. 31, no. 1, pp. 20-22, 2010.

[9] R. Riaza, "Nondegeneracy conditions for active memristive circuits," IEEE Transactions on Circuits and Systems II, vol. 57, no. 3, pp. 223-227, 2010.

[10] I. Petráš, "Fractional-order memristor-based Chua's circuit," IEEE Transactions on Circuits and Systems II, vol. 57, no. 12, pp. 975-979, 2010.

[11] F. Merrikh-Bayat and S. B. Shouraki, "Memristor-based circuits for performing basic arithmetic operations," Procedia Computer Science, vol. 3, pp. 128-132, 2011.

[12] F. Merrikh-Bayat and S. B. Shouraki, "Programming of memristor crossbars by using genetic algorithm," Procedia Computer Science, vol. 3, pp. 232-237, 2011.

[13] S. Shin, K. Kim, and S. M. Kang, "Memristor applications for programmable analog ICs," IEEE Transactions on Nanotechnology, vol. 10, no. 2, pp. 266-274, 2011.

[14] T. Berzina, A. Smerieri, M. Bernab ò et al., "Optimization of an organic memristor as an adaptive memory element," Journal of Applied Physics, vol. 105, no. 12, Article ID 124515, 5 pages, 2009.

[15] H. Li and M. Hu, "Compact model of memristors and its application in computing systems," in Proceedings of the Design, Automation and Test in Europe Conference and Exhibition (DATE '10), pp. 673-678, March 2010.

[16] F. Corinto, A. Ascoli, and M. Gilli, "Nonlinear dynamics of memristor oscillators," IEEE Transactions on Circuits and Systems I, vol. 58, no. 6, pp. 1323-1336, 2011.

[17] G. Cserey, A. Rak, B. Jakli, and T. Prodromakis, "Cellular Nonlinear Networks with Memristive Cell Devices," in Proceedings of IEEE International Conference on Electronics, Circuits, and Systems (ICECS '10), pp. 940-943, December 2010.

[18] Z. Wu, P. Shi, H. Su, and J. Chu, "Exponential synchronization of neural networks with discrete and distributed delays under time-varying sampling," IEEE Transactions on Neural Networks and Learning Systems, vol. 23, pp. 1368-1376, 2012.

[19] Z. Wu, P. Shi, H. Su, and J. Chu, "Delay-dependent stability analysis for switched neural networks with time-varying delay," IEEE Transactions on Systems, Man and Cybernetics B, vol. 41, pp. 1522-1530, 2011.
[20] Z. Wu, P. Shi, H. Su, and J. Chu, "Passivity analysis for discretetime stochastic Markovian jump neural networks with mixed time-delays," IEEE Transactions on Neural Networks, vol. 22, no. 10, pp. 1566-1575, 2011.

[21] X. Luan, P. Shi, and F. Liu, "Robust adaptive control for greenhouse climate using neural networks," International Journal of Robust and Nonlinear Control, vol. 21, no. 7, pp. 815-826, 2011.

[22] Z. G. Wu, P. Shi, H. Su, and J. Chu, "Delay-dependent exponential stability analysis for discrete-time switched neural networks with time-varying delay," Neurocomputing, vol. 74, no. 10, pp. 1626-1631, 2011.

[23] R. Yang, Z. Zhang, and P. Shi, "Exponential stability on stochastic neural networks with discrete interval and distributed delays," IEEE Transactions on Neural Networks, vol. 21, no. 1, pp. 169-175, 2010.

[24] H. Li, H. Gao, and P. Shi, "New passivity analysis for neural networks with discrete and distributed delays," IEEE Transactions on Neural Networks, vol. 21, no. 11, pp. 1842-1847, 2010.

[25] X. Dong, Y. Zhao, Y. Xu, Z. Zhang, and P. Shi, "Design of PSO fuzzy neural network control for ball and plate system," International Journal of Innovative Computing, Information and Control, vol. 7, pp. 7091-7104, 2011.

[26] T. Chien, "Feedforward neural network and feedback linearization control design of BILSAT-1 satellite system," International Journal of Innovative Computig, Information and Control, vol. 8, pp. 6921-6943, 2012.

[27] Y. Fu and T. Chai, "Nonlinear adaptive decoupling control based on neural networks and multiple models," International Journal of Innovative Computing, Information and Control, vol. 8, pp. 1867-1878, 2012.

[28] W. Ding and L. Wang, " $2^{N}$ almost periodic attractors for CohenGrossberg-type BAM neural networks with variable coefficients and distributed delays," Journal of Mathematical Analysis and Applications, vol. 373, no. 1, pp. 322-342, 2011.

[29] Y. Liu, Z. You, and L. Cao, "On the almost periodic solution of generalized Hopfield neural networks with time-varying delays," Neurocomputing, vol. 69, no. 13-15, pp. 1760-1767, 2006.

[30] H. Xiang and J. Cao, "Almost periodic solution of Cohen-Grossberg neural networks with bounded and unbounded delays," Nonlinear Analysis: Real World Applications, vol. 10, no. 4, pp. 2407-2419, 2009.

[31] L. Wang, "Existence and global attractivity of almost periodic solutions for delayed high-ordered neural networks," Neurocomputing, vol. 73, no. 4-6, pp. 802-808, 2010.

[32] C. Bai, "Existence and stability of almost periodic solutions of Hopfield neural networks with continuously distributed delays," Nonlinear Analysis, Theory, Methods and Applications, vol. 71, no. 11, pp. 5850-5859, 2009.

[33] H. Jiang, L. Zhang, and Z. Teng, "Existence and global exponential stability of almost periodic solution for cellular neural networks with variable coefficients and time-varying delays," IEEE Transactions on Neural Networks, vol. 16, no. 6, pp. 13401351, 2005.

[34] L. Wang, W. Lu, and T. Chen, "Multistability and new attraction basins of almost-periodic solutions of delayed neural networks," IEEE Transactions on Neural Networks, vol. 20, no. 10, pp. 1581$1593,2009$.

[35] W. Lu and T. Chen, "Almost periodic dynamics of a class of delayed neural networks with discontinuous activations," Neural Computation, vol. 20, no. 4, pp. 1065-1090, 2008. 
[36] W. Allegretto, D. Papini, and M. Forti, "Common asymptotic behavior of solutions and almost periodicity for discontinuous, delayed, and impulsive neural networks," IEEE Transactions on Neural Networks, vol. 21, no. 7, pp. 1110-1125, 2010.

[37] M. Forti, P. Nistri, and D. Papini, "Global exponential stability and global convergence in finite time of delayed neural networks with infinite gain," IEEE Transactions on Neural Networks, vol. 16, no. 6, pp. 1449-1463, 2005.

[38] H. Wu, "Stability analysis for periodic solution of neural networks with discontinuous neuron activations," Nonlinear Analysis: Real World Applications, vol. 10, no. 3, pp. 1717-1729, 2009.

[39] Z. Cai and L. Huang, "Existence an global asymptotic stability of periodic solution for discrete and distributed time-varying delayed neural networks with discontinuous activations," Neurocomputing, vol. 74, pp. 3170-3179, 2011.

[40] D. Papini and V. Taddei, "Global exponential stability of the periodic solution of a delayed neural network with discontinuous activations," Physics Letters A, vol. 343, no. 1-3, pp. 117-128, 2005.

[41] X. Chen and Q. Song, "Global exponential stability of the periodic solution of delayed Cohen-Grossberg neural networks with discontinuous activations," Neurocomputing, vol. 73, no. 16-18, pp. 3097-3104, 2010.

[42] H. Wu, "Global stability analysis of a general class of discontinuous neural networks with linear growth activation functions," Information Sciences, vol. 179, no. 19, pp. 3432-3441, 2009.

[43] X. Liu and J. Cao, "On periodic solutions of neural networks via differential inclusions," Neural Networks, vol. 22, no. 4, pp. 329-334, 2009.

[44] X. He, W. Lu, and T. Chen, "Nonnegative periodic dynamics of delayed Cohen-Grossberg neural networks with discontinuous activations," Neurocomputing, vol. 73, no. 13-15, pp. 2765-2772, 2010.

[45] H. Wu and C. Shan, "Stability analysis for periodic solution of BAM neural networks with discontinuous neuron activations and impulses," Applied Mathematical Modelling, vol. 33, no. 6, pp. 2564-2574, 2009.

[46] M. Itoh and L. O. Chua, "Memristor cellular automata and memristor discrete-time cellular neural networks," International Journal of Bifurcation and Chaos, vol. 19, no. 11, pp.3605-3656, 2009.

[47] Y. V. Pershin and M. Di Ventra, "Experimental demonstration of associative memory with memristive neural networks," Neural Networks, vol. 23, no. 7, pp. 881-886, 2010.

[48] J. Hu and J. Wang, "Global uniform asymptotic stability of memristor-based recurrent neural networks with time delays," in Proceedings of the International Joint Conference on Neural Networks (IJCNN '10), pp. 1-8, Barcelona, Spain, July 2010.

[49] A. Wu, Z. Zeng, X. Zhu, and J. Zhang, "Exponential synchronization of memristor-based recurrent neural networks with time delays," Neurocomputing, vol. 74, pp. 3043-3050, 2011.

[50] A. Wu, S. Wen, and Z. Zeng, "Synchronization control of a class of memristor-based recurrent neural networks," Information Sciences, vol. 183, pp. 106-116, 2012.

[51] A. Wu, J. Zhang, and Z. Zeng, "Dynamic behaviors of a class of memristor-based Hopfield networks," Physics Letters A, vol. 375, no. 15, pp. 1661-1665, 2011.

[52] S. Wen and Z. Zeng, "Dynamics analysis of a class of memristorbased recurrent networks with time-varying delays in the presence of strong external stimuli," Neural Processing Letters, vol. 35, pp. 47-59, 2012.
[53] J.-P. Aubin and A. Cellina, Differential Inclusions, Springer, Berlin, Germany, 1984.

[54] A. F. Filippov, Differential Equations with Discontinuous Righthand Sides, vol. 18, Kluwer Academic Publishers, Boston, Mass, USA, 1988.

[55] B. M. Levitan and V. V. Zhikov, Almost Periodic Functions and Differential Equations, Cambridge University, Cambridge, UK, 1982.

[56] H. Dong, Z. Wang, and H. Gao, "Distributed filtering for a class of time-varying systems over sensor networks with quantization errors and successive packet dropouts," IEEE Transactions on Signal Processing, vol. 60, no. 6, pp. 3164-3173, 2012.

[57] Z. Wang, Y. Liu, and X. Liu, "Exponential stabilization of a class of stochastic system with markovian jump parameters and mode-dependent mixed time-delays," IEEE Transactions on Automatic Control, vol. 55, no. 7, pp. 1656-1662, 2010.

[58] H. Dong, Z. Wang, J. Jam, and H. Gao, "Fuzzy-model-based robust fault detection with stochastic mixed time delays and successive packet droputs," IEEE Transactions on Systems, Man, and Cybernetics B, vol. 42, pp. 365-376, 2012. 


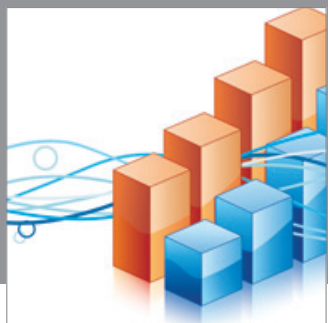

Advances in

Operations Research

mansans

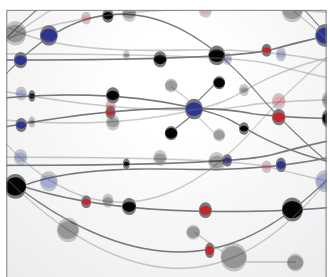

The Scientific World Journal
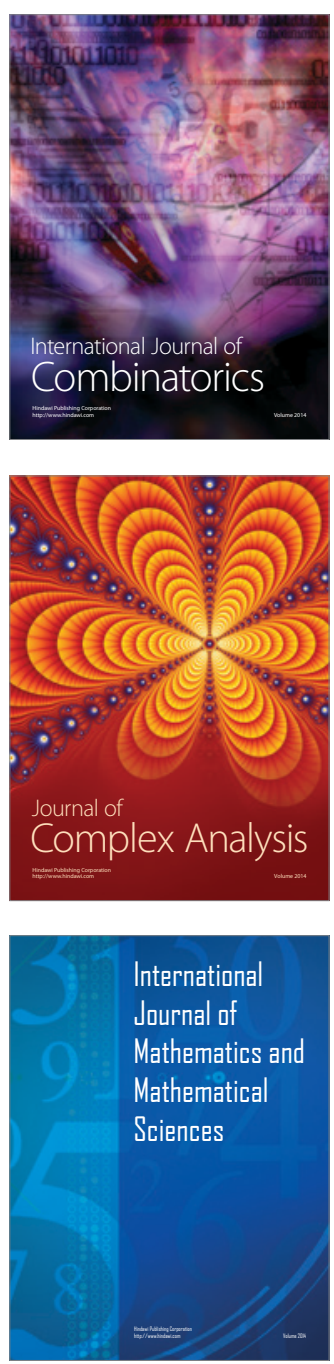
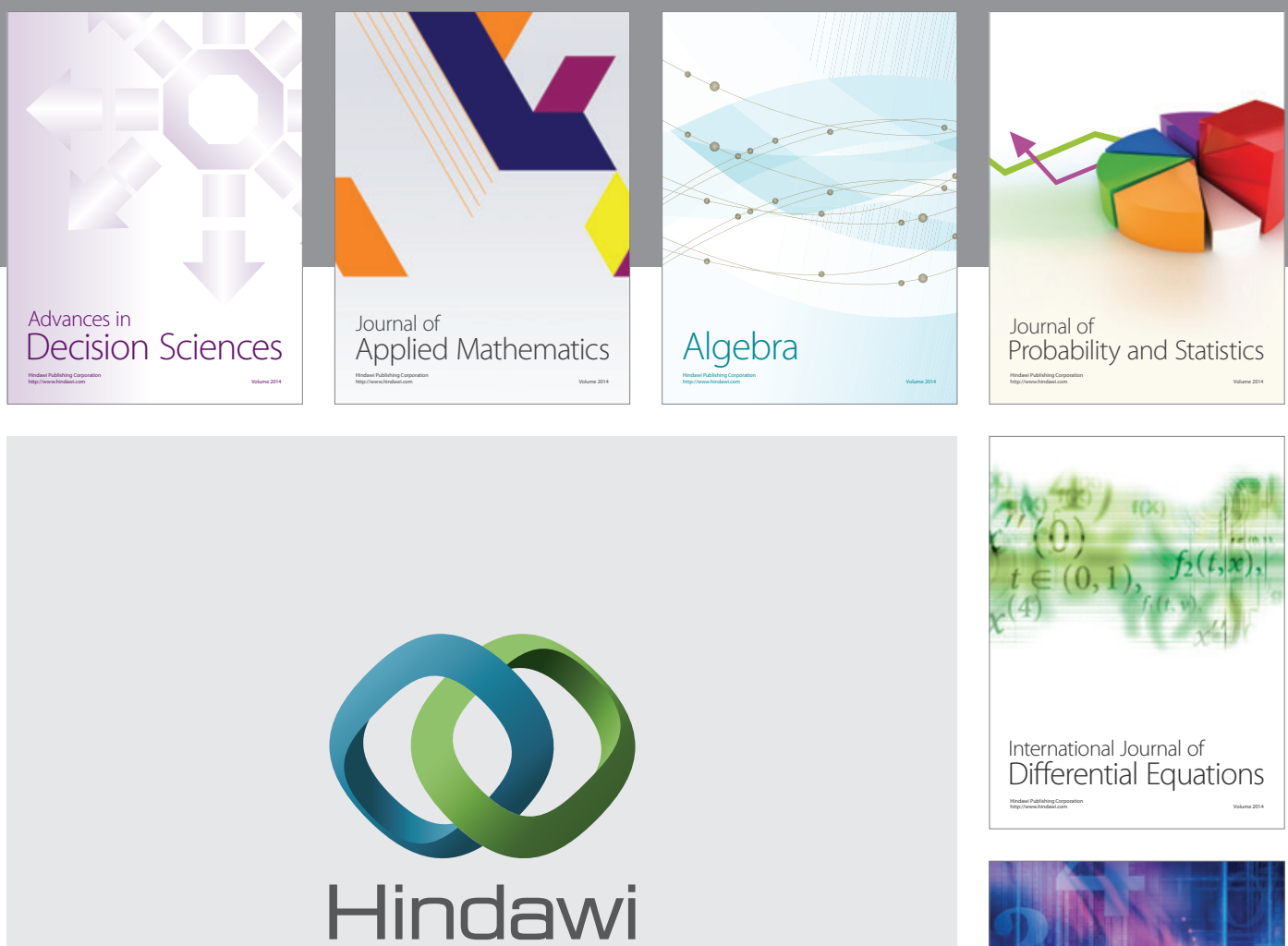

Submit your manuscripts at http://www.hindawi.com
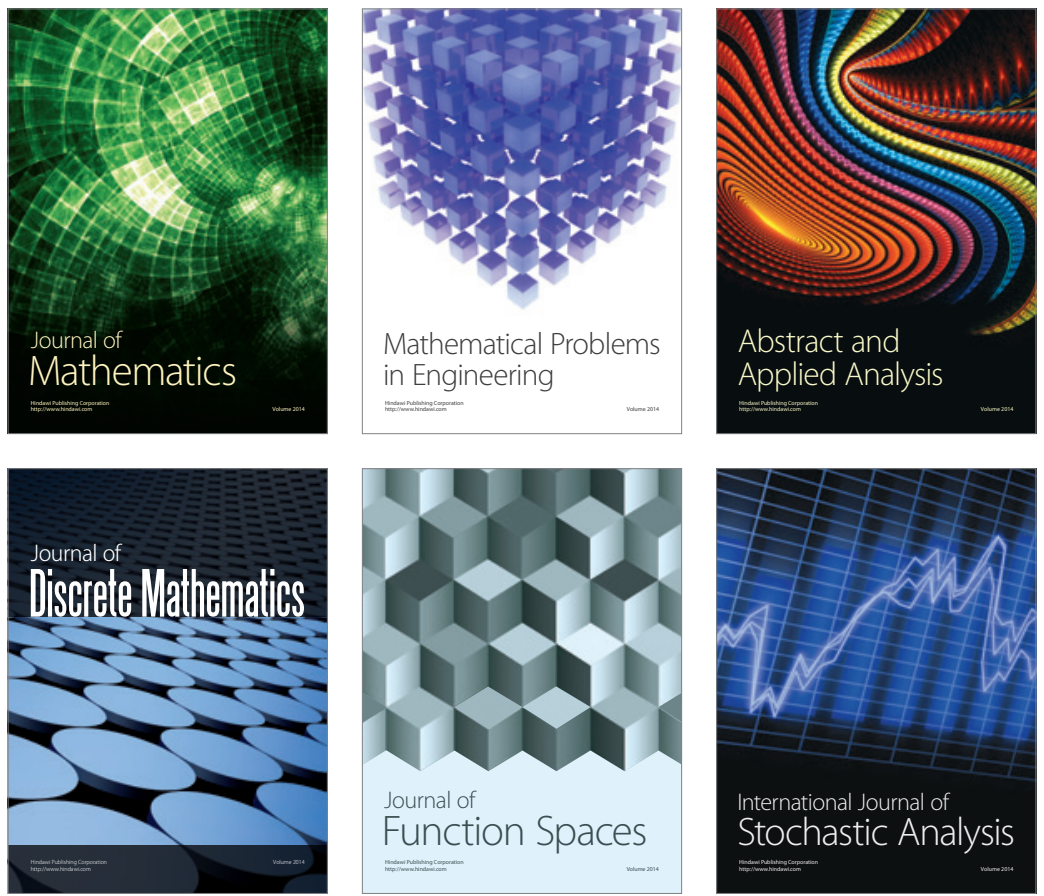

Journal of

Function Spaces

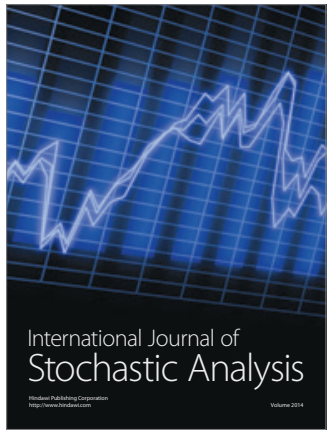

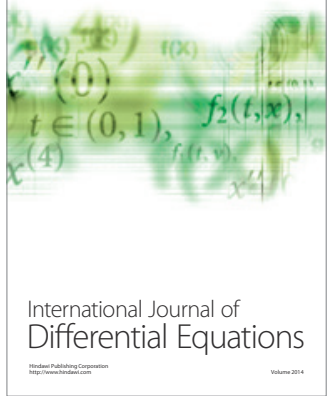
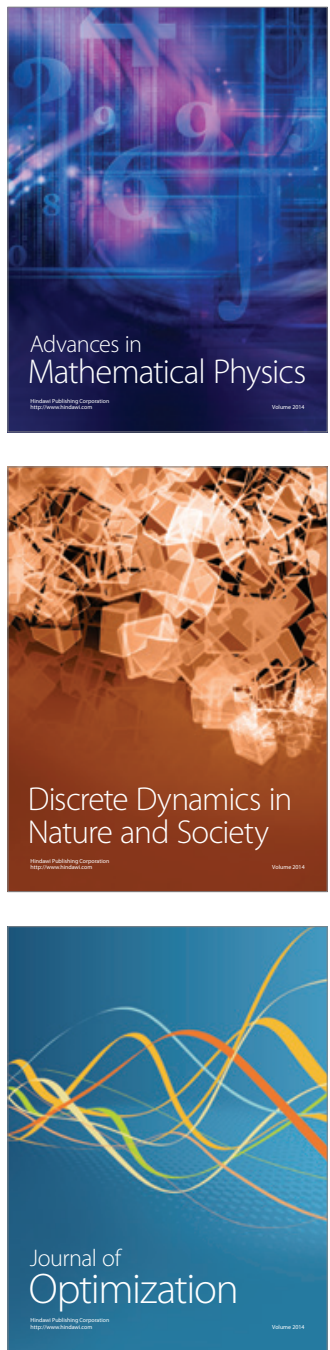